\title{
kW-level, narrow-linewidth linearly polarized fiber laser with excellent beam quality through compact one-stage amplification scheme
}

\author{
Man Jiang ${ }^{1}$, Pengfei $\mathrm{Ma}^{1,2}$, Long Huang ${ }^{1}$, Jiangming $\mathrm{Xu}^{1,2}$, Pu Zhou ${ }^{1,2}$, and Xijia Gu${ }^{3}$ \\ ${ }^{1}$ College of Optoelectronic Science and Engineering, National University of Defense Technology, Changsha 410073, China \\ ${ }^{2}$ Hunan Provincial Collaborative Innovation Center of High Power Fiber Laser, Changsha 410073, China \\ ${ }^{3}$ Department of Electrical and Computer Engineering, Ryerson University, 350 Victoria St., Toronto, Ontario M5B 2K3, Canada \\ (Received 4 June 2017; revised 30 September 2017; accepted 30 October 2017)
}

\begin{abstract}
In this manuscript, we demonstrate high-power, narrow-linewidth linearly polarized fiber laser with excellent beam quality through compact one-stage amplification scheme. By employing a single-mode-multimode-single-mode structure seed laser, a linearly polarized Yb-doped fiber laser with narrow linewidth and high output power is achieved. This laser, when used as a master oscillator, can be capable of suppressing the ASE in the process of power amplification. Thus, only one-stage amplification structure is used to scale up the laser power, and linearly polarized output with a polarization extinction ration of $14 \mathrm{~dB}$, a narrow linewidth of $0.3 \mathrm{~nm}$ and an output power of $1018 \mathrm{~W}$ are achieved. Moreover, due to the good beam quality of seed laser and the well-designed amplifier stage, the beam quality of the output laser is near-diffraction-limited with $M_{x}^{2} \sim 1.18$ and $M_{y}^{2} \sim 1.24$ at the maximum power, and without mode instability occurring.
\end{abstract}

Keywords: fiber amplifier; fiber Bragg grating; linearly polarization; narrow linewidth

\section{Introduction}

The technology of high-power fiber laser has made impressive progress in the last decades - output power of single-mode near-diffraction-limited Yb-doped fiber (YDF) lasers reached $\sim 5 \mathrm{~kW}$ when pumped entirely by diodes and $\sim 10 \mathrm{~kW}$ with the amplifier in-band tandem pumping ${ }^{[1,2]}$. However, further output power scale-up faces some major obstacles coming from fiber nonlinearities, transverse mode instability (MI) and optical damage, which are very challenging to overcome. Many research groups have been studying alternative techniques to scale up laser power; among them, spectral beam combining (SBC) stands out as a promising one to achieve high brightness laser source ${ }^{[3-7]}$.

Due to the spectral dispersion of the dispersive elements used in the SBC such as multilayer dielectric gratings ${ }^{[3,4]}$ or volume Bragg gratings ${ }^{[5]}$, the linewidth of each individual beam has to be reasonably narrow in order to endure the quality of the combined beam. Therefore, a narrowlinewidth YDF laser has attracted considerable attention

Correspondence to: P. Zhou, College of Optoelectronic Science and Engineering, National University of Defense Technology, Changsha 410073, China. Email: zhoupu203@163.com for further brightness scaling. In Ref. [3], 96 channels of $\sim 300 \mathrm{~W}$ fiber lasers were combined into one single beam of $30-\mathrm{kW}$ output power, which is the typical milestone in high-power SBC. Power scaling should be expected if the output power of each element could be increased to $\mathrm{kW}$ level. In fact, Zheng et al. reported an eight-element fiber laser SBC system that achieved $10.8 \mathrm{~kW}$, and each channel were $1.5 \mathrm{~kW}^{[6]}$. However, three-stage YDF amplifier chains were used in order to boost the output power of the narrowband laser seed, that made the individual laser element complicated. To obtain high-power narrow-linewidth laser source, many works were based on cascaded multi-stage power amplification scheme to boost the output power ${ }^{[8-11]}$. Moreover, in an SBC system, a polarization-independent multilayer dielectric diffraction grating had been used and each individual laser element was randomly polarized with a diffraction efficiency of $94 \%{ }^{[6]}$, while the diffraction efficiency of the polarization-dependent multilayer dielectric grating can be as high as $97 \%^{[12]}$. Thus, one can increase the combined efficiency by employing the linearly polarized laser array. Therefore, high-power linearly polarized fiber laser channel with compact structure would optimize the performance of SBC system. 
In order to develop more simple and compact designs to achieve both narrow linewidth and high power, many research groups have demonstrated the methods based on master oscillator power amplifier (MOPA) structure to scale up the output power of narrow-linewidth laser oscillator directly.

Hao et al. reported an all-fiber narrow-linewidth amplifier seeded by a laser oscillator by use of a pair of extremely narrow-bandwidth fiber Bragg gratings (FBGs). The 13$\mathrm{dB}$ reflection bandwidth was 0.1 and $0.05 \mathrm{~nm}$ for the high reflective (HR) FGB and the output coupler (OC) FGB, respectively ${ }^{[13]}$. Although a narrow linewidth of $0.08 \mathrm{~nm}$ at an output power of $823 \mathrm{~W}$ had been obtained, a cavity requiring such precision wavelength matching is difficult to maintain since a temperature change of $0.1^{\circ} \mathrm{C}$ will result in a wavelength change of $1 \mathrm{pm}$ for an $\mathrm{FBG}^{[14]}$. Thus, a few degree temperature offset between the two FBGs would deteriorate the cavity's Q-factor substantially.

The method to achieve narrow-linewidth output was also demonstrated by shortening the gain fiber lengths for both oscillator and amplifier. Very recently, Huang et al. demonstrated a $2.9 \mathrm{~kW}$ YDF amplifier with an output linewidth of $60 \mathrm{pm}$ for the seed laser and $310 \mathrm{pm}$ for the amplifier ${ }^{[15]}$ Another attempt to achieve both narrow linewidth and high power was reported by using a master oscillator of $\sim 25 \mathrm{GHz}$ (96 pm) at $6 \mathrm{~W}$ output power and obtained $\sim 75 \mathrm{GHz}$ (286 pm) linewidth at $2 \mathrm{~kW}$ output ${ }^{[16]}$. Due to the insufficient power of the seed laser, two-stage amplifiers were used to suppress the amplified spontaneous emission (ASE) effectively. This design, despite the success, was complicated the same as the aforementioned cascaded multi-stage power amplification scheme. If a high-power narrow-linewidth laser source based on single-stage power-scaling scheme can be demonstrated, it would relatively simplify the laser system and reduce the costing ${ }^{[17,18]}$. Besides, the outputs of those three reports mentioned before were not linearly polarized.

In this paper, we use a single-stage amplifier to scale linearly polarized fiber laser to $\mathrm{kW}$ level while maintaining a narrow spectral linewidth. Our results show that this MOPA scheme achieved a linearly polarized laser output with average power of $1018 \mathrm{~W}$ and a narrow linewidth of $0.3 \mathrm{~nm}$. Moreover, due to the good beam quality of seed laser and the well-designed amplifier stage, the output laser has a near-diffraction-limited beam quality with $M_{x}^{2} \sim 1.18$ and $M_{y}^{2} \sim 1.24$ at the maximum power without MI occurring.

\section{Experiments}

Figure 1 shows a schematic diagram of the $1 \mathrm{~kW}$ narrow spectral width all-fiber amplifier structure. The MOPA configuration consisted of a CW fiber laser oscillator and a double cladding fiber amplifier.

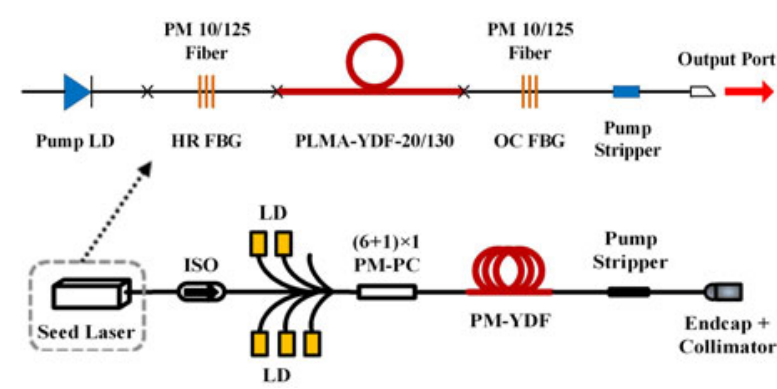

Figure 1. Experimental setup of the narrow-linewidth, linearly polarized seed laser and fiber amplifier. LD: laser diode; PC: polarization controller; ISO: isolator.

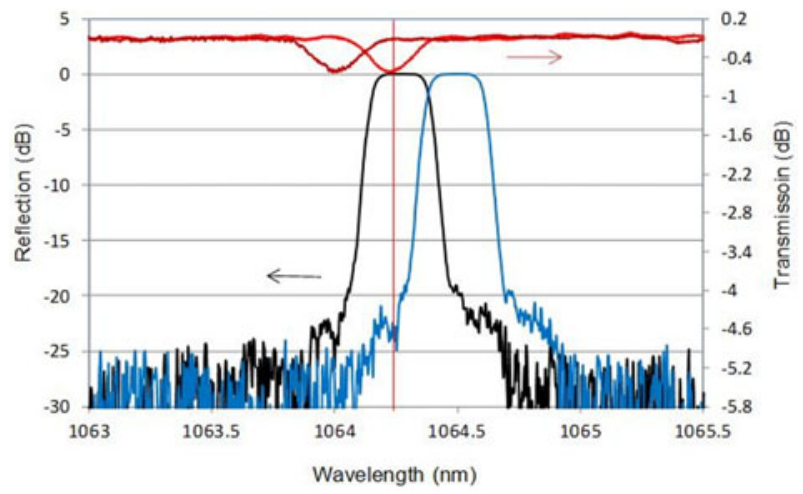

Figure 2. Reflection spectra of the HR-FBG and transmission spectra of OC-FBG on fast and slow axis, respectively.

The oscillator is a linear cavity laser, consisting of a pair of FBGs and a piece of gain YDF. The FBG with an HRFBG of $99 \%$ at $1064 \mathrm{~nm}$ is spliced to one end of the gain fiber with the axes of both fiber aligned. The OC-FBG with a reflectivity of $10 \%$ is spliced to the other end of the gain fiber after a $90^{\circ}$ rotation. The spectra of the HR-FBG and OC-FBG, as shown in Figure 2, are shifted for the purpose of polarization selection: fast axis wavelength of the HR-FBG $(1064.226 \mathrm{~nm})$ matches with the slow axis wavelength of the OC-FBG $(1064.251 \mathrm{~nm})$ within a tolerance of $30 \mathrm{pm}$. The full width at half maximum (FWHM) bandwidth of the HRFBG is $0.235 \mathrm{~nm}$ and $0.17 \mathrm{~nm}$ for OC-FBG, respectively.

Another design is employed in the cavity in order to reduce the intensity in the core of gain fiber and thus reduce self-phase modulation (SPM) effect. A $1.3 \mathrm{~m}$ long 20/130 $\mu \mathrm{m}$ multimode YDF (PLMA-YDF-20/130, Nufern Inc.) with a nominal cladding absorption coefficient of $10.2 \mathrm{~dB} / \mathrm{m}$ at $976 \mathrm{~nm}$ is used as the gain fiber and two pieces of $10 / 125 \mu \mathrm{m}$ fiber (PLMA-GDF-10/125, Nufern Inc.) are used to inscribe FBGs; thus the cavity consists of an single-mode-multimode-single-mode (SMS) design. The SMS design was used in Refs. [19-22] before which suppressed Raman emission and reduced SPMinduced linewidth broadening. However, in this design we attempted to use polarization-maintaining (PM) fibers to 

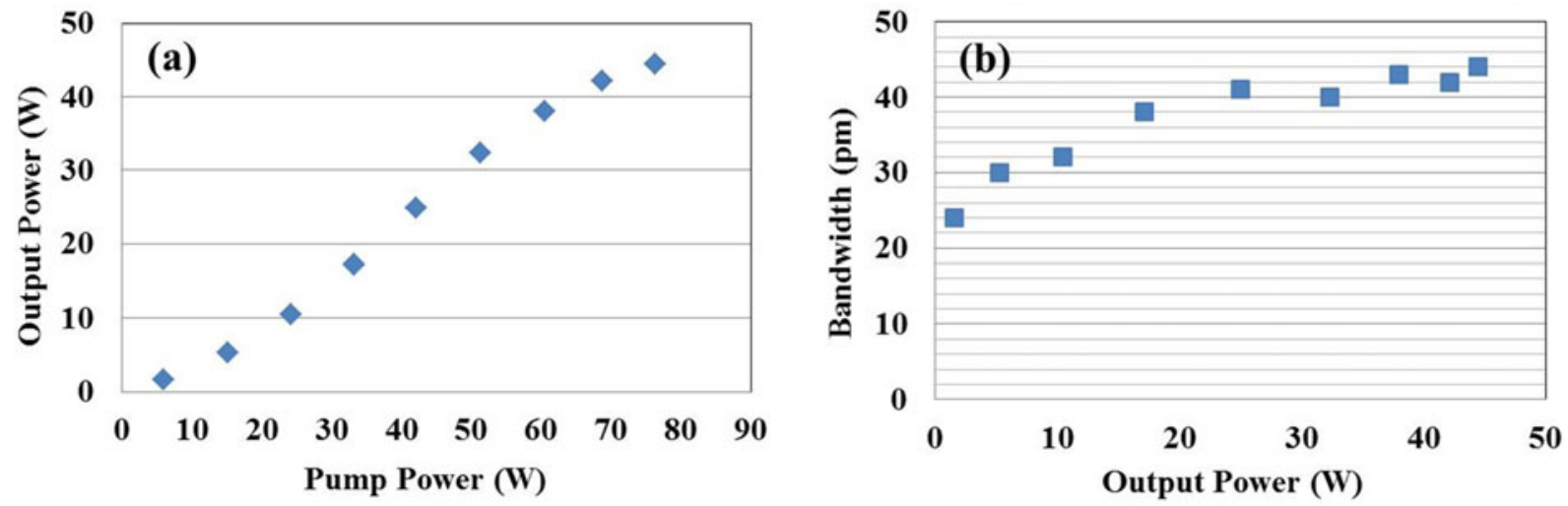

Figure 3. Master oscillator: (a) laser output power versus pump power; (b) spectral linewidth at different output power.

construct an SMS cavity. Moreover, we use larger mode area gain fiber by comparison with Ref. [19] to see if it would increase power conversion efficiency, meanwhile reducing SPM effect which would broaden the laser linewidth.

A 90-W $976 \mathrm{~nm}$ fiber-coupled multimode laser diode (MMLD) is utilized to end-pump the active fiber. The residual pump light and some signal laser propagating in the fiber cladding are leaked out by using a power stripper at the output end of the oscillator. At the rear end ports of the oscillator, a PM isolator is incorporated into the MOPA structure to block off the backward powers from the following amplification, the backward ASE for example, to protect the oscillator. The seed oscillator can produce output power of $\sim 45 \mathrm{~W}$ with slope efficiency of $\sim 64.8 \%$, as shown in Figure 3(a), and further power scaling would be achieved by increasing pump power and adding hydrocooling. The $-3 \mathrm{~dB}$ bandwidth of output emission spectra measured at different output power were $\leqslant 44 \mathrm{pm}$ as shown in Figure 3(b). One can see that the slope efficiency of this laser is less than that in Ref. [19] (with slope efficiency of $\sim 68.5 \%$ ), due to the splicing loss between 20/130 $\mu \mathrm{m}$ YDF and $10 / 125 \mu \mathrm{m}$ fiber is larger than that between $15 / 130 \mu \mathrm{m}$ YDF and $10 / 125 \mu \mathrm{m}$ fiber. However, the output spectral widths in this paper become narrower compared to Ref. [19], in which the $-3 \mathrm{~dB}$ bandwidth started at $42 \mathrm{pm}$ and increased up to $52 \mathrm{pm}$ at $32.7 \mathrm{~W}$. Due to the large mode area (LMA) gain fiber which would reduce the SMP effect in laser cavity, the seed laser in this paper obtains a higher output power, meanwhile maintaining the linewidth narrower. Moreover, the polarization extinction ratio (PER) at maximum output power is measured to be $>15 \mathrm{~dB}$. Due to such performance of this laser, when used as a master oscillator, it should be capable of scaling up the output power to multi-kW level by a single-stage amplifier while maintaining a narrow spectral linewidth.

The power amplifier is pumped by five wavelengthstabilized, $300 \mathrm{~W}$ power-level laser diodes (LDs) with $976 \mathrm{~nm}$ central wavelength via a $(6+1) \times 1$ PM pump combiner. The active fiber in this stage is LMA and double clad PM YDF with a core diameter of $20 \mu \mathrm{m}$ and an inner cladding diameter of $400 \mu \mathrm{m}$ (PLMA-YDF-20/400). The cladding absorption coefficient is about $1.7 \mathrm{~dB} / \mathrm{m}$ at $976 \mathrm{~nm}$ and an $8.5 \mathrm{~m}$ long active fiber is employed for high-power scaling. It is to be noted that in this amplifier, in order to suppress MI effect effectively and maintain good beam quality, the active fiber is coiling with radius of $\sim 5.5 \mathrm{~cm}^{[10,23]}$. About $1 \mathrm{~m}$ long PM passive fiber and a high-power fiber end-cap with $\sim 1.5 \mathrm{~m}$ long PM passive fiber are successively fused to the rear end of the active fiber for power delivering. The core and inner cladding diameters of the two pieces of passive fibers remain the same as the active fiber. In the $1 \mathrm{~m}$ long passive fiber, an $\sim 40 \mathrm{~cm}$ highindex gel section is made for stripping out the residual pump and cladding light. The delivering laser through the fiber end-cap is collimated into free space by using a high-power beam collimator.

\section{Results and Discussions}

Output power of this amplifier as a function of pump power is measured within $20 \mathrm{~W}$ seed laser injection. At $1336 \mathrm{~W}$ coupled pump power, up to $1018 \mathrm{~W}$ laser output is obtained with an overall slope efficiency $\sim 78.6 \%$, as shown in Figure 4.

An optical spectrum analyzer (ANDO Corp. model AQ6317) with $0.01 \mathrm{~nm}$ resolution is used to measure the spectral characteristics of the output laser. The spectrum at the maximum output power is plotted in Figure 5, which shows that the center wavelength is located at $1064.36 \mathrm{~nm}$ and an optical signal to noise ratio of $\sim 40 \mathrm{~dB}$ is achieved. No residual power at $976 \mathrm{~nm}$ and ASE spectra is observed.

A close-up view of the output laser spectra is shown in Figure 6, in which, the optical signal-noise ratio (OSNR) is about $35 \mathrm{~dB}$ across all output power levels. After the onestage fiber amplifiers, the $-3 \mathrm{~dB}$ bandwidth and $-10 \mathrm{~dB}$ bandwidth of the laser emission spectra are measured, respectively, at different output powers as shown in Figure 7. Due to the nonlinear effects including SPM and four-wave 


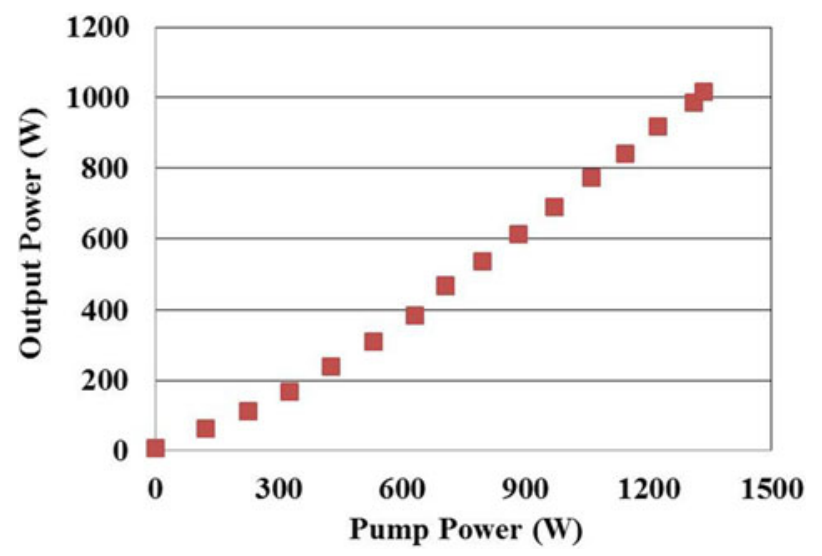

Figure 4. Laser output power versus pump power.

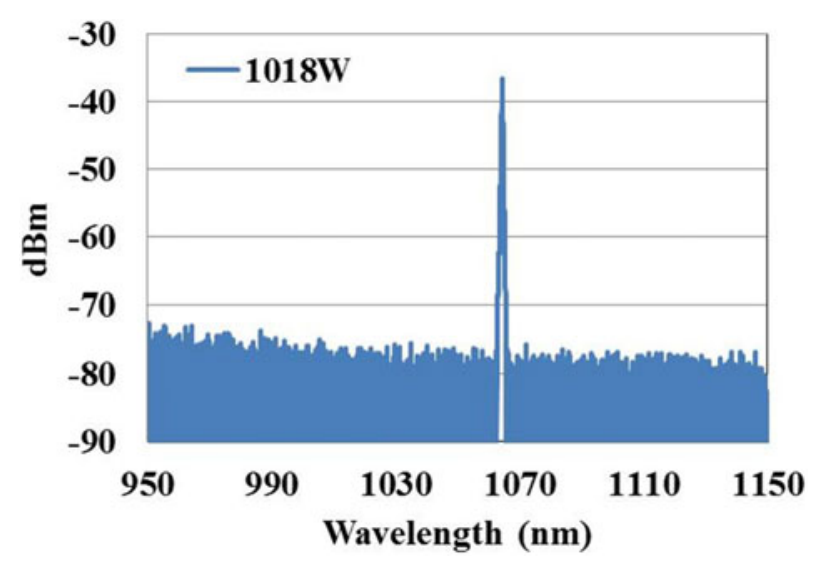

Figure 5. Optical spectrum at $1018 \mathrm{~W}$ maximum laser.

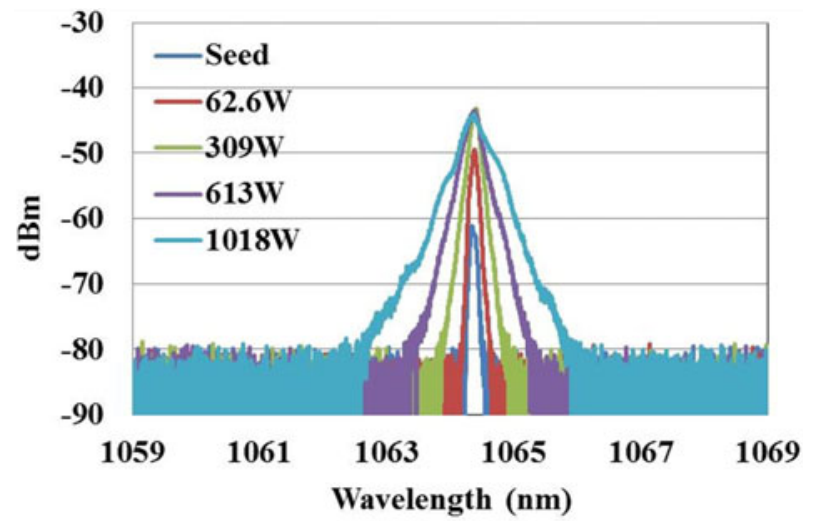

Figure 6. An enlarged view of the laser spectrum at different output power.

mixing (FWM), the $-3 \mathrm{~dB}$ bandwidth starts at $0.1 \mathrm{~nm}$ and increases up to $0.3 \mathrm{~nm}$ at $1018 \mathrm{~W}$, meanwhile the corresponding $-10 \mathrm{~dB}$ bandwidth increases from $0.2 \mathrm{~nm}$ to $0.9 \mathrm{~nm}$. By increasing injection seed power or adding pump power would enhance the output power of the amplifier.

The PER is measured with a high-quality polarization beam splitter (PBS). The laser power transmitted through,

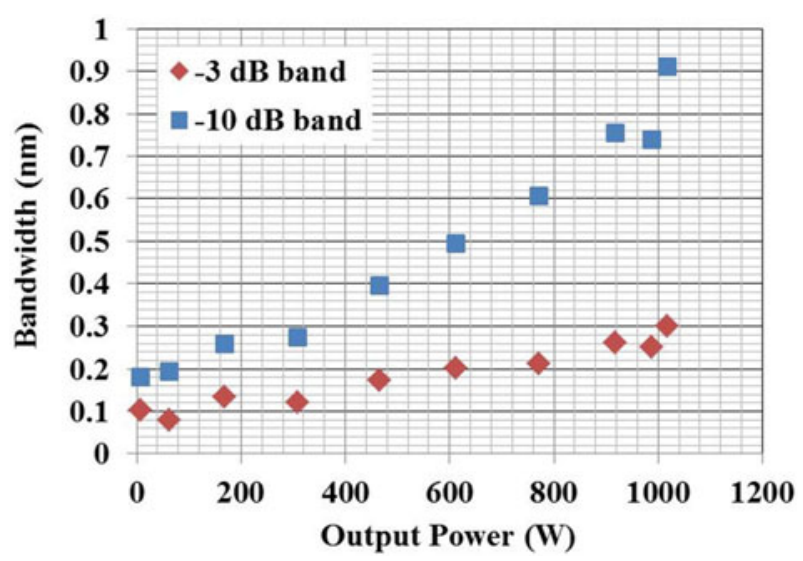

Figure 7. Spectral linewidth as a function of laser power.

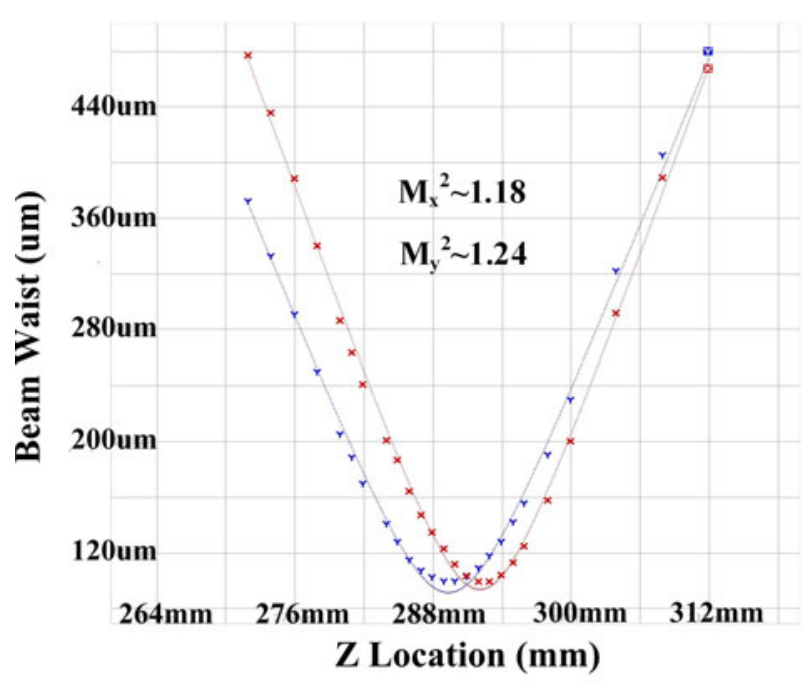

Figure 8. Beam quality factor $\left(M^{2}\right)$ of the output laser beam at $1018 \mathrm{~W}$.

and reflected by the PBS is measured consecutively when the power from one polarization is minimized by rotating the collimated laser beam. The PER is obtained from the ratio of the two power readings at different output power. The measured PER is reached $14 \mathrm{~dB}$ at maximum output power.

In addition, the well-designed PM amplifier which is operated stably contributes to the excellent beam quality of the kW-level amplifier output beam. As Figure 8 shows, using a beam profile analyzer $\left(M^{2}-200\right)$ the beam quality factor $M^{2}$ is measured to be $\sim 1.18$ and $\sim 1.24$ in the $x$ and $y$ directions respectively, for the laser beam with $1018 \mathrm{~W}$ output power. Nearly diffraction-limited beam quality is achieved.

\section{Conclusions}

In summary, we have successfully demonstrated a kW-level linearly polarized laser source with narrow linewidth and 
near-diffraction-limited beam quality based on a compact power-scaling configuration. By employing a high-power SMS structure seed laser, only one-stage amplification is used to scale up the output power to $1018 \mathrm{~W}$ with a slope efficiency of $\sim 78.6 \%$. It has the linearly polarized output with a PER of $14 \mathrm{~dB}$, and a narrow linewidth of below $0.3 \mathrm{~nm}$ is obtained. Due to the good beam quality of seed laser and the well-designed amplifier stage, the beam quality of the output laser is near-diffraction-limited with $M_{x}^{2} \sim 1.18$ and $M_{y}^{2} \sim 1.24$ without $\mathrm{MI}$ occurring at the maximum power. The output power of the laser amplifier could still be enhanced by increasing injection seed power or adding pump power. It is worth mentioning that the principle of our design is general and can be applied to other wavelengths of the fiber amplifier. This all-fiber single-stage amplification construction of the whole MOPA system enables compact size and robust operation. In addition, the narrow-linewidth linearly polarized high-power amplifiers are also desirable for many important applications such as beam combining, lidar, laser materials processing, etc.

\section{Acknowledgements}

This work was supported by Huo Ying Dong Education Foundation of China (No. 151062) and the Foundation for the author of National Excellent Doctoral Dissertation of China (No. 201329).

\section{References}

1. W. Shi, Q. Fang, and Y. Qin, in SPIE Photonics West (2015).

2. M. N. Zervas and C. A. Codemard, IEEE J. Sel. Top. Quantum Electron 20, 0904123 (2014).

3. E. Honea, R. S. Afzal, M. S.-Leuchs, J. Henrie, K. Brar, N. Kurz, D. Jander, N. Gitkind, D. Hu, C. Robin, A. M. Jones, R. Kasinadhuni, and R. Humphreys, Proc. SPIE 9730, 97300Y (2016).
4. C. Wirth, O. Schmidt, I. Tsybin, T. Schreiber, R. Eberhardt, J. Limpert, A. Tünnermann, K. Ludewigt, M. Gowin, E. ten Have, and M. Jung, Opt. Lett. 36, 3118 (2011).

5. D. Ott, I. Divliansky, B. Anderson, G. Venus, and L. Glebov, Opt. Express 21, 29620 (2013).

6. Y. Zheng, Y. Yang, J. Wang, M. Hu, G. Liu, X. Zhao, X. Chen, K. Liu, C. Zhao, B. He, and J. Zhou, Opt. Express 24, 12064 (2016).

7. P. Ma, M. Jiang, X. Wang, Y. Ma, P. Zhou, and Z. Liu, IEEE Photon. Technol. Lett. 27, 2099 (2015).

8. N. A. Naderi, I. Dajani, and A. Flores, Opt. Lett. 41, 1018 (2016).

9. C. X. Yu, O. Shatrovoy, T. Y. Fan, and T. F. Taunay, Opt. Lett. 41, 5202 (2016).

10. P. Ma, R. Tao, R. Su, X. Wang, P. Zhou, and Z. Liu, Opt. Express 24, 4187 (2016).

11. L. Huang, H. Wu, R. Li, L. Li, P. Ma, X. Wang, J. Leng, and P. Zhou, Opt. Lett. 42, 1 (2017).

12. T. Clausnitzer, J. Limpert, K. Zöllner, H. Zellmer, H.-J. Fuchs, E.-B. Kley, A. Tünnermann, M. Jupé, and D. Ristau, Appl. Opt. 42, 6934 (2003).

13. J. Hao, H. Zhao, and D. Zhang, Appl. Opt. 54, 4857 (2015).

14. A. D. Kersey, M. A. Davis, H. J. Patrick, M. Leblanc, K. P. Koo, C. G. Askins, M. A. Putnam, and E. J. Friebele, J. Lightwave Technol. 15, 1442 (1997).

15. Z. Huang, X. Liang, C. Li, H. Lin, Q. Li, J. Wang, and F. Jing, Appl. Opt. 55, 297 (2016).

16. Y. Xu, Q. Fang, Y. Qin, X. Meng, and W. Shi, Appl. Opt. 54, 9419 (2015)

17. J. Xu, H. Xiao, J. Leng, H. Zhang, P. Zhou, and J. Chen, Laser Phys. Lett. 13, 105101 (2016).

18. C. Yang, S. Xu, S. Mo, C. Li, Z. Feng, D. Chen, Z. Yang, and Z. Jiang, Opt. Express 21, 12546 (2013).

19. M. Jiang, H. Xu, P. Zhou, G. Zhao, and X. Gu, Appl. Opt. 55, 6121 (2016).

20. J. Zhou, B. He, Y. Feng, and X. Gu, Appl. Opt. 53, 5554 (2014).

21. J. Zhou, Y. Lu, B. He, and X. Gu, Appl. Opt. 54, 6080 (2015).

22. M. Jiang, P. Zhou, H. Xiao, and P. Ma, High Power Laser Sci. Eng. 3, e25 (2015).

23. R. Tao, P. Ma, X. Wang, P. Zhou, and Z. Liu, Photon. Res. 3, 86 (2015). 